\title{
Toughening mechanisms in a multi-phase alloy of nylon 6,6/polyphenylene oxide
}

\author{
HUNG-JUE SUE, ALBERT F. YEE \\ Macromolecular Research Center and Department of Materials Science and Engineering, \\ University of Michigan, Ann Arbor, Michigan 48109, USA
}

Toughening mechanisms in a nylon 6,6/polyphenylene oxide (PA/PPO) alloy are studied. This alloy consists of well dispersed PPO particles (containing an elastomeric phase) in a PA matrix. Both crazing and shear yielding mechanisms are found to be sequentially operative in this alloy. When a crack propagates in the material, a crazed zone forms ahead of the crack tip. This crazed zone then transforms into a shear yielded zone as the crack propagates through it. The crazes inside the original crazed zone are closed or distorted by the shear yielding process. In the transformed region, the shear yielded material is oriented at different angles as the crack propagates. These findings are consistent with the notion that hydrostatic tension has to be dissipated in order for the remaining deviatoric stress to reach the critical value for yielding. It is possible to partially attribute the increase in toughness to both crack blunting and zone shielding mechanisms.

\section{Introduction}

Recent developments in multi-phase alloys of two or more rigid* polymers [1-10] have led to a new generation of materials. Examples of such alloys are polybutylene terephthalate/polycarbonate, nylon 6,6/ polyphenylene oxide (PA/PPO) and acrylonitrilebutadiene-styrene/polycarbonate. These rigid-rigid polymer alloys benefit from desirable combinations of mechanical and physical properties of the constituents. Depending on the composition, however, the rigidrigid polymer alloys may not always be tough. In fact, mixing two or three randomly chosen rigid polymers will usually result in blends with poor and unpredictable properties. To assure that toughened alloys are obtained consistently, it is imperative that we understand the specific material parameters which are responsible for unleashing all potential toughening mechanisms that can operate in rigid-rigid polymer alloys. The present paper examines the toughening mechanisms in a commercial PA/PPO alloy using various techniques. The current study is part of a larger effort to gain insight into the toughening mechanisms of a new generation of engineering polymers, i.e., rigid-rigid polymer alloys. Questions regarding mechanics, interfaces, rheology and processing will be addressed in future publications.

To increase the toughness of a given material, the material needs to possess effective energy absorbing mechanisms. For thermoplastics, it appears that massive crazing and massive shear yielding are the most frequently encountered energy absorbing mechanisms. In the case of massive crazing, the toughener phase must have good adhesion with the matrix phase [11]. Moreover, depending on the matrix material, the size of the toughening particle is thought to exert a tremendous effect on the maximum toughness [12]. The toughener phase serves not only as a stress concentrator but also as a craze stabilizer $[12,13]$. In general, the massive-crazing type mechanism increases the notched Izod impact toughness of the unmodified polymer only several-fold $[11,13]$. On the other hand, if the matrix polymer is toughened via the shear yielding mechanism, the toughener phase apparently needs only meet two requirements: (i) relieve the triaxial tension and (ii) generate a localized stress concentration [14]. Furthermore, (i) and (ii) must occur sequentially, as we attempt to demonstrate in this paper. When these conditions are met, an order of magnitude increase in toughness may be obtained [14]. Within the range 0.1 to $10 \mu \mathrm{m}$ the particle size does not seem to affect significantly the toughness via shear yielding $[14,15]$.

When a specimen possessing a crack is loaded by applying a far field tensile stress perpendicular to the crack, a state of triaxial tension exists ahead of the crack tip. The shear yield stress for polymers is pressure sensitive, but the effect is small. Thus even though the hydrostatic tension will reduce the yield stress somewhat the effect is not large enough to cause shear yielding significantly earlier to occur. Instead, the triaxial tension, if not dissipated, will eventually reach a critical value for craze-crack formation which, in turn, will lead to brittle failure. As a result, dissipation of the triaxial tension is critical for toughening a polymer under a constrained condition. The relief of the triaxial tension will facilitate shear yielding because the deviatoric stress remains high [14]. Shear yielding will create a plastic zone, thus blunting the crack. A

* Rigid means non-elastomeric in the context of this paper. 
number of mechanisms, such as cavitation of the toughener phase, debonding at the interface between the toughener phase and the matrix, and generation of massive crazing, can serve to dissipate the tri-axial tension. So far, however, only the cavitation of the toughener phase has been examined in detail [14-23]. In rigid-rigid polymer alloys, cavitation of the toughener (dispersed) phase may not be a viable mechanism for dissipation of triaxial tension. In fact, owing to the typically small difference between the elastic constants of the two constituents, debonding at the interface between the toughener phase and the matrix, as well as generation of massive crazes, are more likely to occur in rigid-rigid polymer alloys.

Moreover, to effect toughening, a certain degree of interfacial adhesion between the matrix and the dispersed phase is critical. It is still not known, however, how strong the adhesion between the matrix and the dispersed phase should be in order for the toughness of an alloy to be optimized. It is known that poor adhesion between the phases can cause debonding at the interface upon the application of a finite load. This event can either nucleate cracking or crazing or initiate the onset of localized shear deformation, depending on the polymer. With a strongly adhering interface, both phases share and transmit the load, leading to undiminished stiffness in the system. Without interfacial failure, some other cavitational mechanism must take place to relieve the hydrostatic tension.

In this work, a number of techniques, such as tensile dilatometry, fractography, and fracture using single edge notched three point bending (SEN-3PB) specimens, scanning electron microscopy (SEM), transmission electron microscopy (TEM), and transmitted light optical microscopy (TOM), were employed to investigate the PA/PPO alloy. In addition, a double notched four-point bending (DN-4PB) specimen was used to probe the fracture behaviour of the PA/PPO alloy at the crack initiation and propagation stages. The study of the fractured DN-4PB specimen using TOM and TEM techniques has proven to be very useful. We report in this paper information obtained from these studies concerning the possible toughening mechanisms in the PA/PPO alloy.

\section{Experimental procedure}

The PA/PPO alloy, known commercially as GTX 910, was obtained from the General Electric Company. According to the manufacturer's literature, this material comprises $50 \%$ by weight $\mathrm{PA}$ and $50 \%$ by weight PPO modified with an undisclosed amount of elastomeric material. Prior to the fabrication process, the material in pellet form was dried in a vacuum oven for $4 \mathrm{~h}$ at $65^{\circ} \mathrm{C}$. Several types of specimens were prepared for this study. These included ASTM standard tensile specimens (D638, type I), a tapered tensile specimen, $1 / 4^{\prime \prime}(6.35 \mathrm{~mm})$ thick SEN$3 \mathrm{~PB}$ specimens, and $1 / 4^{\prime \prime}$ thick DN-4PB specimens. Except for the tapered tensile specimens, all specimens were injection moulded. The injection moulded specimens were stored in ambient conditions for several months prior to testing. It can be assumed that the PA contains the equilibrium amount of water $(\approx 3 \mathrm{wt} \%)$ for room temperature.

\subsection{Tensile dilatometry}

Tensile dilatometry, a technique made popular by Bucknall and coworkers [13] was shown by them to be effective in deciphering the types of micromechanisms that operate during the deformation of specimens in tension. It is particularly useful for distinguishing between dilatative and volume-conserving deformation events. In the present experiments a computer controlled servo-hydraulic tensile testing machine (Instron model 1332) was used to perform the tensile tests at constant cross-head speeds. Two highly sensitive extensometers were employed for each tensile dilatometric test to measure the longitudinal and transverse (width) strains. The thickness strain is assumed to be the same as the width strain. This assumption, though approximate, is thought to be adequate to indicate the deformation mechanisms. The volume strain is calculated using the following formula

$$
\frac{\Delta V}{V_{0}}=\left(1+\varepsilon_{1}\right)\left(1+\varepsilon_{\mathrm{t}}\right)^{2}-1
$$

where $\Delta V$ is the change in volume, $V_{0}$ the original volume, and $\varepsilon_{1}$ and $\varepsilon_{\mathrm{t}}$ are the longitudinal and transverse engineering strains, respectively. Since $\varepsilon_{1}$ is usually less than 0.1 and $\varepsilon_{\mathrm{t}}$ is smaller still, the formula determines true volume strain to a very good approximation. Under these conditions, the $\sigma-\varepsilon-\Delta$ (i.e., stress-strain-dilatation) behaviour of the PA/PPO alloy was measured. The measurement was continued until evidence of necking appeared in the specimen. A detailed description of the technique can be found in a previous publication [14]. This technique can measure volume changes with a resolution of $0.02 \%$.

\subsection{Tapered tensile specimen}

The tensile dilatometry results turned out to be very complex. In order to understand the dilatation behaviour of the PA/PPO alloy, an injection moulded D638 type I tensile specimen was tapered to the dimensions shown in Fig. 1. Upon loading, the stress amplitude on the tapered tensile specimen varied along the entire gauge length.

Our previous experience indicates that a higher testing strain rate will raise the stress for craze initiation, while the strain where crazing begins increases with increasing strain rate [24]. With a slower applied strain rate enough time is allowed for many crazes to form uniformly [24]. Therefore, in order to avoid any possible localized craze formation, the specimen was tested using a screw driven tensile testing machine (Instron model 1137) at a low crosshead speed of $0.05^{\prime \prime} \mathrm{min}^{-1}$ $\left(0.021 \mathrm{~mm} \mathrm{sec}^{-1}\right)$. The testing machine was stopped when the narrowest section began to show significant stress-whitening. The tested specimen, which had previously been marked into divisions, was cut into several blocks. The stress amplitude within each block was calculated from the dimensions of the tapered tensile specimen and the maximum applied load.

The blocks were polished to remove the skin region. The remaining cores were then stained with a $2 \%$ 


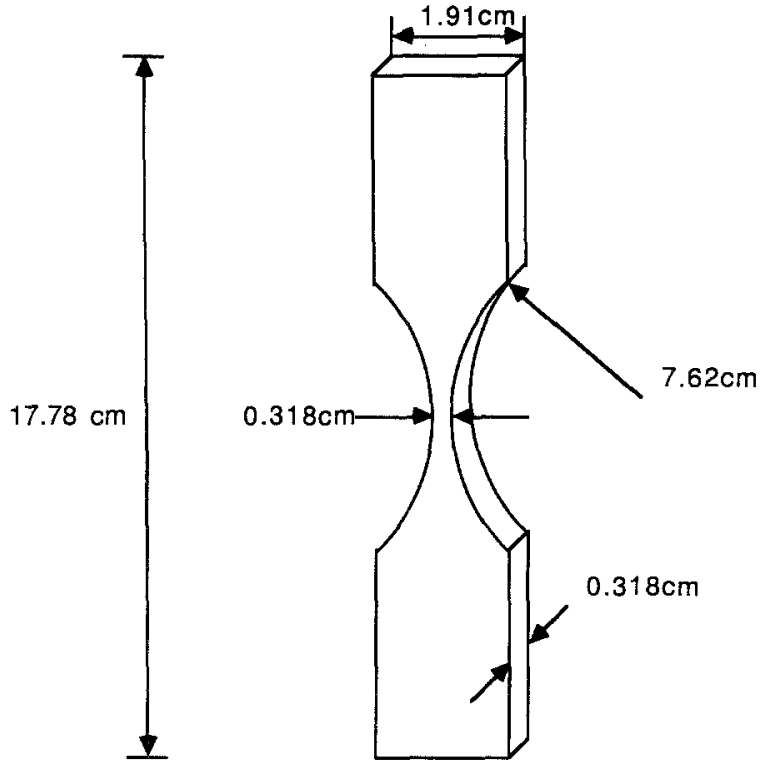

Figure 1 A schematic drawing of the tapered tensile specimen. The specimen was cut from an injection moulded ASTM standard tensile specimen (D638 type I). The radius of curvature of the tapered specimen is $7.62 \mathrm{~cm}$.

aqueous solution of $\mathrm{OsO}_{4}$ for about $48 \mathrm{~h}$. Afterwards, these blocks were microtomed using a Reichert Ultracut E43 to make thin sections on the order of $0.1 \mu \mathrm{m}$ in thickness. The sections are parallel to the stress direction. The thin sections were laid on top of copper grids and examined using a Zeiss EM-10A (or Philips 400) TEM operating at an accelerating voltage of $100 \mathrm{kV}$.

\subsection{Fractographic studies}

SEN-3PB and DN-4PB specimens of PA/PPO were prepared from injection moulded bars of dimensions $5 / 2^{\prime \prime} \times 1 / 2^{\prime \prime} \times 1 / 4^{\prime \prime}(63.5 \mathrm{~mm} \times 12.7 \mathrm{~mm} \times 6.35 \mathrm{~mm})$. The notch was made by first cutting with a diamond saw to give an appropriate notch length and then with a fresh razor blade which had been cooled in liquid nitrogen to ensure a sharp crack tip. The cold blade was tapped lightly into the saw cut with a hammer to wedge open a crack ahead of the blade tip. In the case of the double-notched specimen efforts were made to ensure that the cracks thus generated were as nearly equal in length as possible. The geometry of these specimens is sketched in Fig. 2. The specimens were tested at $20^{\prime \prime} \mathrm{min}^{-1}\left(8.47 \mathrm{~mm} \mathrm{sec}^{-1}\right)$ using a screw driven tensile testing machine (Instron model 1137). The fracture surface of the SEN-3PB specimen was coated with a $20 \mathrm{~nm}$ layer of $\mathrm{Au}-\mathrm{Pd}$ for SEM studies.

The purpose of using the DN-4PB specimens is to probe the deformation behaviour of a material around a crack tip before the crack becomes unstable. The material within the two inner loading points in the four-point bending test is subjected to a uniform bending moment. As a result, the two cracks must experience the same stress state if the crack lengths are the same. Since the two cracks were generated at a distance far greater than the plastic zone size of the material due to the crack $(0.4 \mathrm{~cm}$ as opposed to $0.05 \mathrm{~cm}$ ), we can assume that there is no interaction between the two cracks. In the tested DN-4PB specimens the cracks propagated through only one of the

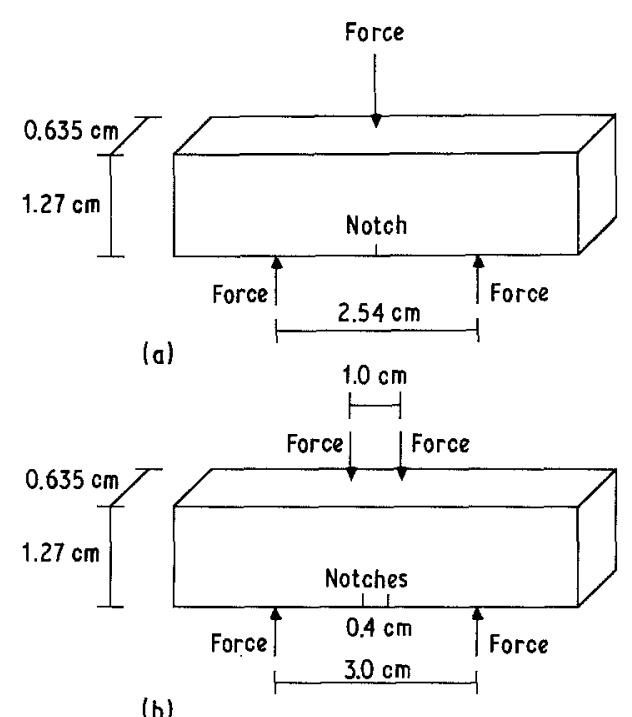

Figure 2 Schematic drawings of (a) the SEN-3PB and (b) DEN-4PB specimens. The specimens were injection moulded into dimensions of $6.35 \mathrm{~cm} \times 1.27 \mathrm{~cm} \times 0.635 \mathrm{~cm}$.

two notches. Thus the unbroken notch preserves information about the plastic zone generated just prior to unstable crack propagation.

Thin $30 \mu \mathrm{m}$ films of PA/PPO were obtained by polishing, following the procedure described by Holik et al. [25]. Thin sections were taken from the midsection (plane strain region) of the fractured SEN-3PB specimens as well as from the mid-section of the unbroken notch tip of the DN-4PB specimens. The sections were made normal to the fracture surface but parallel to the cracking direction. The thin films were then studied using a Nikon Microphot optical microscope.

The tested SEN-3PB and DN-4PB specimens were cut for the TEM studies to a proper block size in a section normal to the fracture surface but parallel to the cracking direction. These blocks were then treated in the same fashion as the procedures previously described for the tapered tensile specimens in the TEM investigations (see above).

\section{Results}

3.1. Tensile dilatometry

Stress-strain and $\varepsilon-\Delta$ data for the PA/PPO alloy were

\section{PA/PPO}

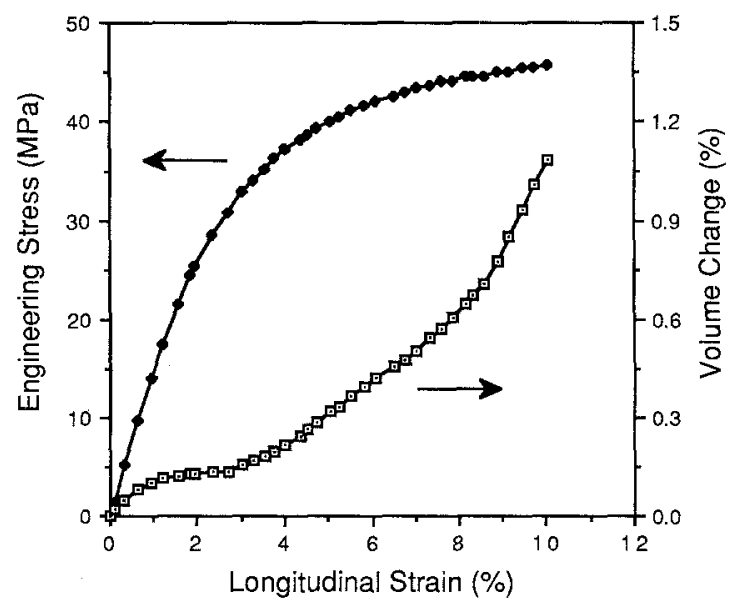

Figure 3 Tensile dilatometry results at a cross-head rate of $0.254 \mathrm{~mm} \mathrm{sec}^{-1}$. 

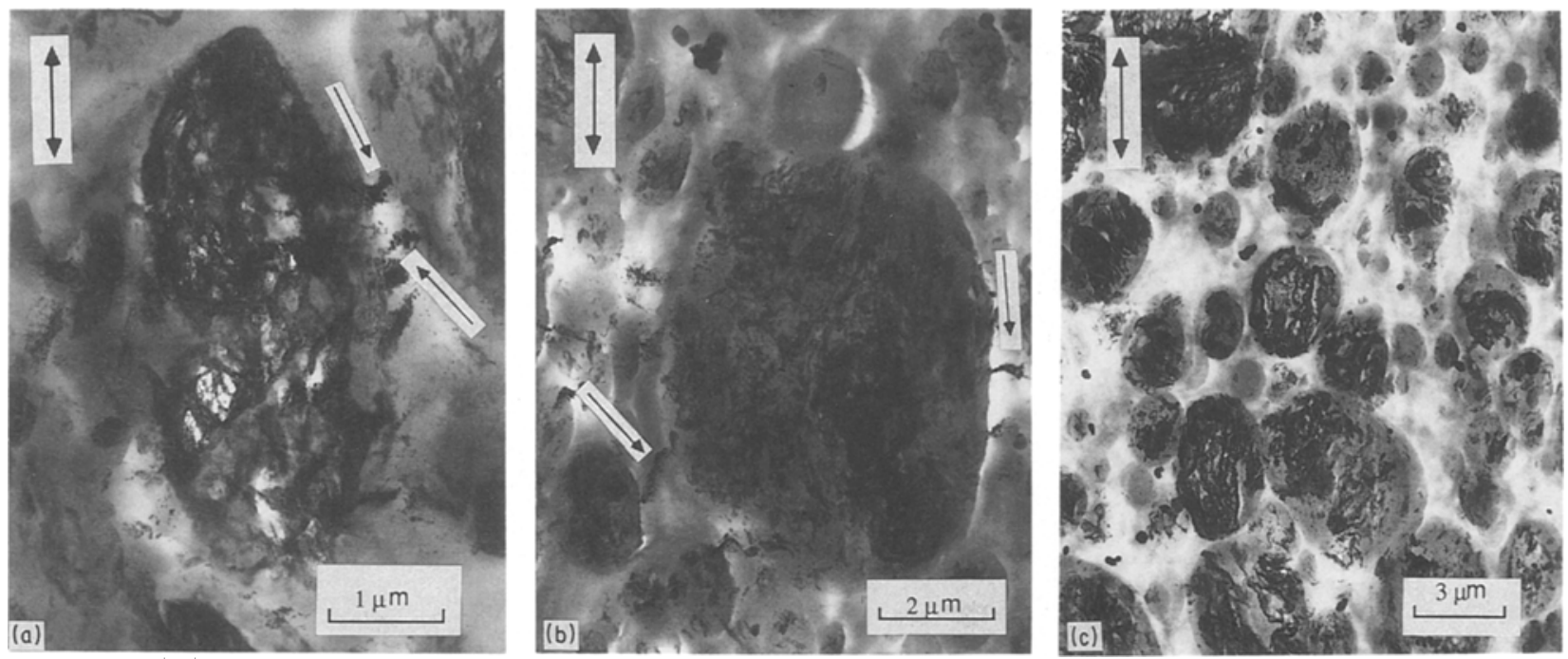

Figure 4 TEM micrographs of the tested tapered tensile specimen. In (a), the micrograph shows evidence of craze formation (see arrows) at the stress-whitening zone; in (b), crazes (see arrows) may still be seen at a section corresponding to a tensile stress state of $\sim 6000 \mathrm{psi}$ ( $40 \mathrm{MPa}$ ); in (c), no craze is found at a section having a tensile stress state less than 5000 psi. Note that the PPO particles have been deformed along the tensile direction as indicated by the double arrow.

obtained simultaneously during the uniaxial tensile tests. The tests were run in duplicate. For convenience, the $\sigma-\varepsilon$ and $\varepsilon-\Delta$ curves are plotted together (Fig. 3). The stress is calculated based on the original value of the cross-sectional area. The stress-strain curve of the PA/PPO alloy does not exhibit a stress drop upon yielding. This behaviour may be due to the moisture absorbed by the PA matrix prior to testing.

An examination of the volume dilatation curve indicates that the deformation process in the PA/PPO alloy is somewhat complex: beyond the initial volume increase due to the elastic response (Poisson's effect) of the sample, the increase in volume slows down at $\sim 1 \% \varepsilon_{1}$; at $\sim 2.5 \% \varepsilon_{1}$ the volume increase begins anew. At $\sim 8.5 \% \varepsilon_{1}$ the slope increases substantially. This complicated volume dilatational behaviour of PA/PPO was examined in greater detail using a tapered tensile specimen.

\subsection{Tapered tensile specimen}

The purpose of using a specimen of this type is to examine the causes for the increase in volume dilation slope at particular stress levels.

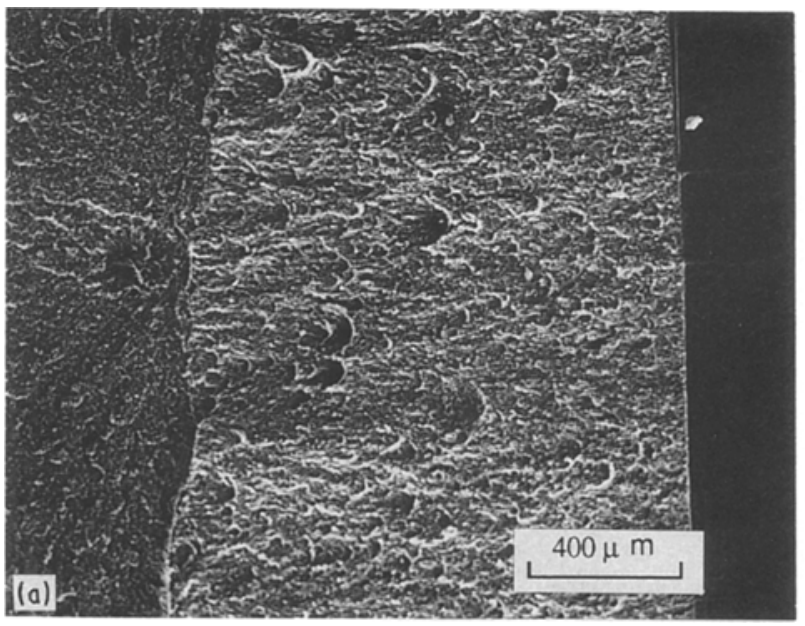

Sections were initially obtained for TOM but we were unable to resolve the crazing features despite the appearance of uniform stress whitening at the maximum stress level. We then prepared ultramicrotomed sections to be studied by TEM. The results indicate that the increase in the volume dilatation slope is due mainly to the formation of crazes, as shown in Fig. 4. The crazes were found to be present in sections corresponding to about $6000 \mathrm{psi}(40 \mathrm{MPa})$ and higher in tensile stress (Figs $4 a$ and $b$ ). No crazes were observed in the PA matrix (Fig. 4c) at stress levels lower than 6000 psi despite exhaustive efforts. We could not ascertain definitively if crazes occurred inside the PPO particles. In all cases the PPO particles were elongated in the tensile direction.

\subsection{Fractographic studies}

The SEN-3PB PA/PPO specimens were tested at $20^{\prime \prime} \min ^{-1}\left(8.47 \mathrm{~mm} \mathrm{sec}^{-1}\right)$. The fracture surfaces near the notch tips contain so-called stress-whitened zones. SEM studies on these stress-whitened regions reveal the widespread formation of cavities along with largescale plastic deformation (see Fig. 5). The size of the

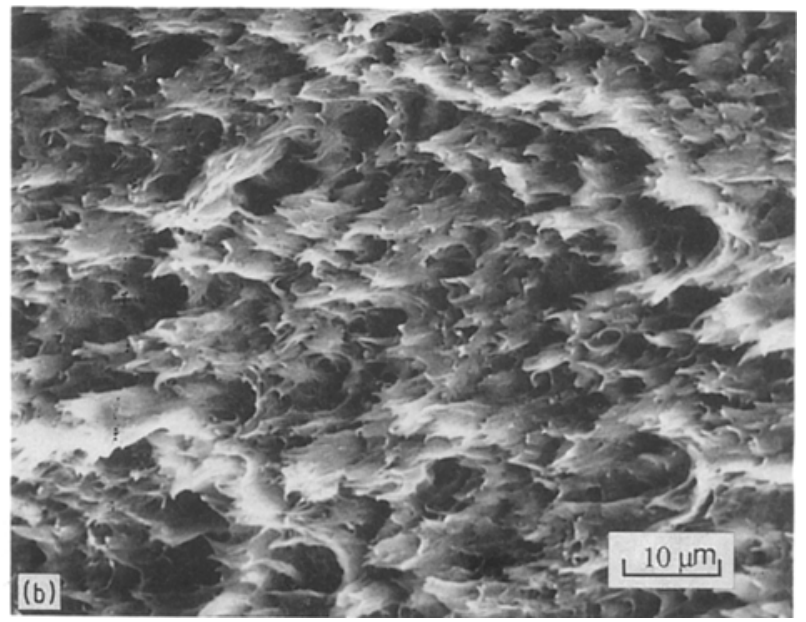

Figure 5 SEM micrographs of the fracture surface of the SEN specimen taken in the stable crack propagation regime. (a) At a low magnification, showing the stress-whitened zone; (b) higher magnification view of the stress-whitened zone. Evidently, large-scale plastic deformation has occurred along with widespread cavitation. The crack propagates from right to left. 

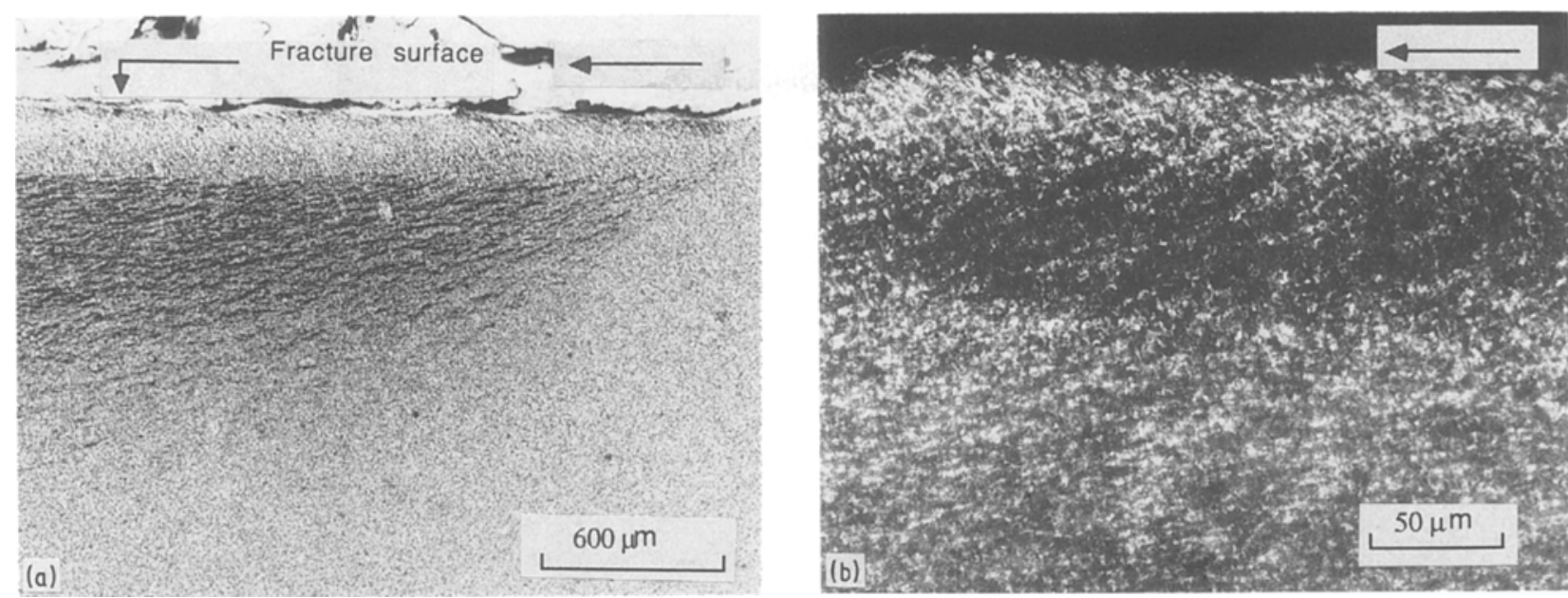

Figure 6 Transmitted light micrographs taken (a) in bright field and (b) between crossed polarizers (at a higher magnification) of the SFSZ of SEN specimen. Massive shear bands are evident immediately beneath the fracture surface. Cavitations, existing as massive crazing, also are observed. The arrow indicates the crack propagation direction.

stress-whitened zone varies with the applied strain rate. With a lower applied strain rate, a larger stresswhitened zone is obtained. This plastic deformation is more easily observed using TOM techniques.

Investigations of thin sections perpendicular to the fracture surface, using TOM techniques, indicate that a birefringent zone due to massive shear banding occurred in the sub-fracture-surface zone (SFSZ), as shown in Fig. 6. Careful inspection of the micrographs reveals that cavitation in the form of crazes occurred as well. In between the shear banding zone and the crazing zone, there exists a region that apparently exhibits neither of these features. These features will be further discussed when the DN-4PB specimens are described.

The unusual features of the SFSZ in the SEN-3PB specimen were further studied using TEM techniques. Figs 7 and 8 indicate that both shear banding and crazing coexist in this plastic zone but in different strata. The micrograph shown in Fig. 7 was taken from the region immediately beneath the fracture surface. Highly elongated PPO particles are found. Assuming that the original undeformed particles are spherical, the estimated strain for these highly elongated particles is about $200 \%$. These particles, in association with the surrounding PA matrix material, must have shear yielded. The crazed zone in the micrographs shown in Fig. 8 reveal three distinguishable phases: (i) a dark elastomeric phase exhibiting a lamella morphology inside the PPO particles; (ii) a grey PPO phase, roughly $3 \mu \mathrm{m}$ in size; and (iii) a white unstained PA matrix phase. Crazes, which are stained particularly well by $\mathrm{OsO}_{4}$ as indicated by the dark horizontal bands, appear to initiate from the elastomeric phase inside the PPO (Fig. 8b). The PPO particles inside the crazed zone were only moderately deformed. In the region farther beneath the fracture surface but above the crazed zone (shown in Fig. 9) the crazes have grown in a somewhat different fashion than those in the crazed zone. These crazes appear to be finer and shorter than those located in the crazed zone. In order to understand how both shear banding and crazing coexist in the SFSZ, DN-4PB specimens were used to study the crack initiation and propagation stages.

As shown in Fig. 10, the optical micrographs of the $\mathrm{DN}-4 \mathrm{~PB}$ specimen clearly indicate that craze zones are formed in front of the crack tip as well as surrounding the shear yielded zone. No shear yielding is apparent in these crazed zones. A close look at the crack tip reveals that a small shear yielded zone is present immediately in front of the crack tip. The existence of this yielded zone ahead of the crack tip is confirmed by the large deformation of the PPO particles adjacent to the crack tip revealed by TEM (see below). Within the region between the starter crack (indicated by the arrow in Fig. 10) and the tip of the crack, a pair of symmetric birefringent zones, i.e., shear yielded zones, are present on each side of the crack. By horizontally rotating the thin section with respect to the crossed polarizers, the apparently featureless region surrounded by the birefringent zone directly adjacent to the crack surface and the birefringent arc is found to contain birefringence, as well (Fig.

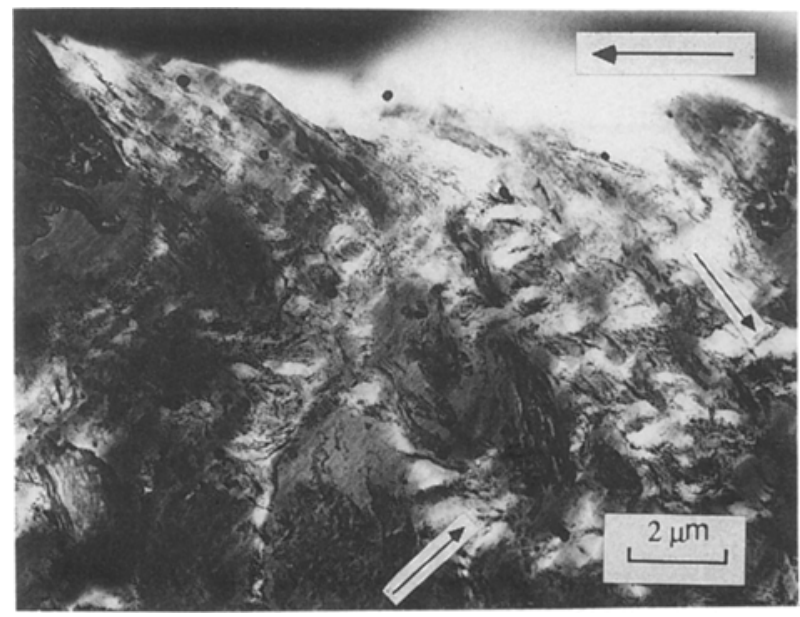

Figure 7 TEM micrograph of a SEN-3PB thin section obtained from the region immediately beneath the fracture surface (upper region of micrographs in Fig. 6). Highly elongated PPO particles were found, suggesting the existence of shear bands. The arrow indicates the crack propagation direction. Small arrows indicate remnants of crazes. 

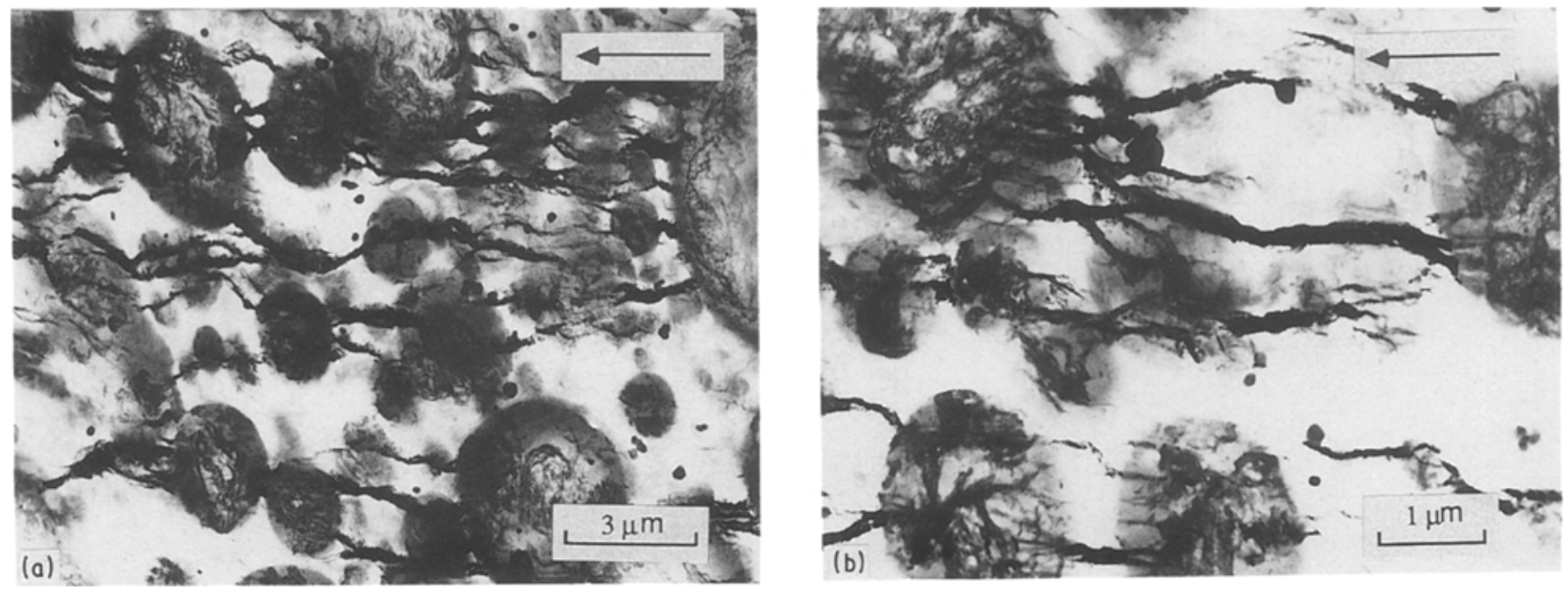

Figure 8 TEM micrographs of a SEN-3PB thin section obtained from the region further beneath the fracture surface (lower region of micrographs in Fig. 6). In (a), at a lower magnification, crazes are observed. As shown in (b), these crazes appear to initiate from the elastomer phase inside the PPO phase. The arrow indicates the crack propagation direction.

11). The amount of birefringence is dependent on the angle between the crack and the polarizers. This phenomenon will be discussed in the next section. These interesting features, which are similar to those observed in the micrographs of the SEN-3PB specimens, were further studied using TEM. As shown in Fig. 12a, highly deformed PPO particles were observed immediately beneath the fracture surface. Very short crazes are also visible in the region farther beneath the fracture surface, Fig. 12b. These crazes have been closed or distorted by the shear yielding. In the crazed zone, Fig. 12c, crazes similar to those observed in the SEN-3PB specimen are found. The crazes in front of the crack tip appear to be somewhat diffuse (Fig. 12d). Farther ahead, at the tip of the crazed zone (Fig. 12e) sharper crazes are formed; the PPO particles here appear to be spherical, similar to those in the undeformed regions. An overview of the deformation at the crack tip is shown in Fig. 13. The crack tip has a radius of curvature comparable to the spacing between the PPO particles which is about $0.5 \mu \mathrm{m}$. The crack appears to propagate directly ahead, along the PA/PPO interface, though a few short side crack branches are

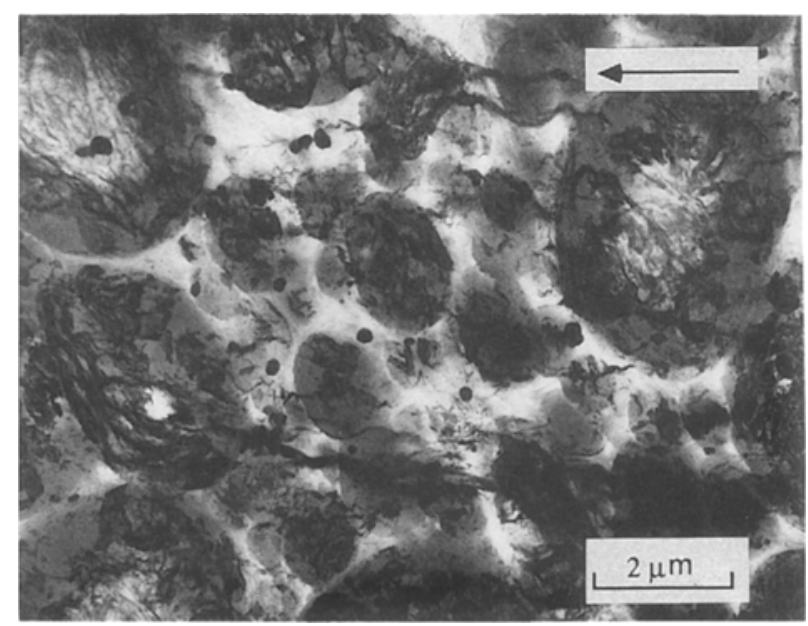

Figure 9 TEM micrograph obtained in the featureless region inside the SFSZ of the SEN-3PB specimen (see Fig. 6). In this micrograph, the crazes appear to be finer and shorter than those in the craze zone. This phenomenon may be due to the closing up or distortion of the crazes. The arrow indicates the crack propagation direction. present. The deformed PPO particles tend to orient themselves according to the stress field along the crack tip. Immediately in front of the crack tip, PPO particles deform to an elongated shape. Since the original PPO particles in the PA/PPO alloy are approximately spherical, the strain in the PPO particles is suggested by the deformation of the spheres. We calculate this strain to be about $60 \%$. Therefore, the material adjacent to the crack tip must have shear yielded despite the fact that profuse crazing has occurred both in the PPO particles and in the matrix surrounding them.

A schematic drawing of the features obtained from the tested DN-4PB specimen is shown in Fig. 14. The TEM micrographs shown in Fig. 12 are indicated in Fig. 14, as well. A summary of the toughening mechanisms in PA/PPO alloy observed in this study is depicted in Fig. 15 for clarity.

\section{Discussion}

The present study concerns the qualitative aspects of the toughening mechanisms in a PA/PPO alloy. It should be noted that this commercial alloy has a very complex composition. It is not possible to definitively determine what roles the individual constituents play in the toughening process. Neither is it possible to unambiguously identify the deformation sequence each constituent exhibits. Nevertheless, it is possible to discuss, in a general way, the components needed to effect toughening.

We have discussed and summarized the details of toughening principles for polymers in our previous work [14] with rubber toughened epoxies. We also have indicated that rigid polymer particles can toughen rigid polymers [1]. The present paper demonstrates another route to toughened polymer systems, i.e., via crazing and transformation of the crazes into shear yielding.

We note at the outset that the patent literature indicates that manufacturers made special efforts to promote adhesion between the PA and PPO phases by using maleic anhydride, fumaric acid, or other reactive agents during the melt compounding [26], presumably to form copolymers of PA and PPO. We surmise that the intention was to promote interfacial adhesion. The 

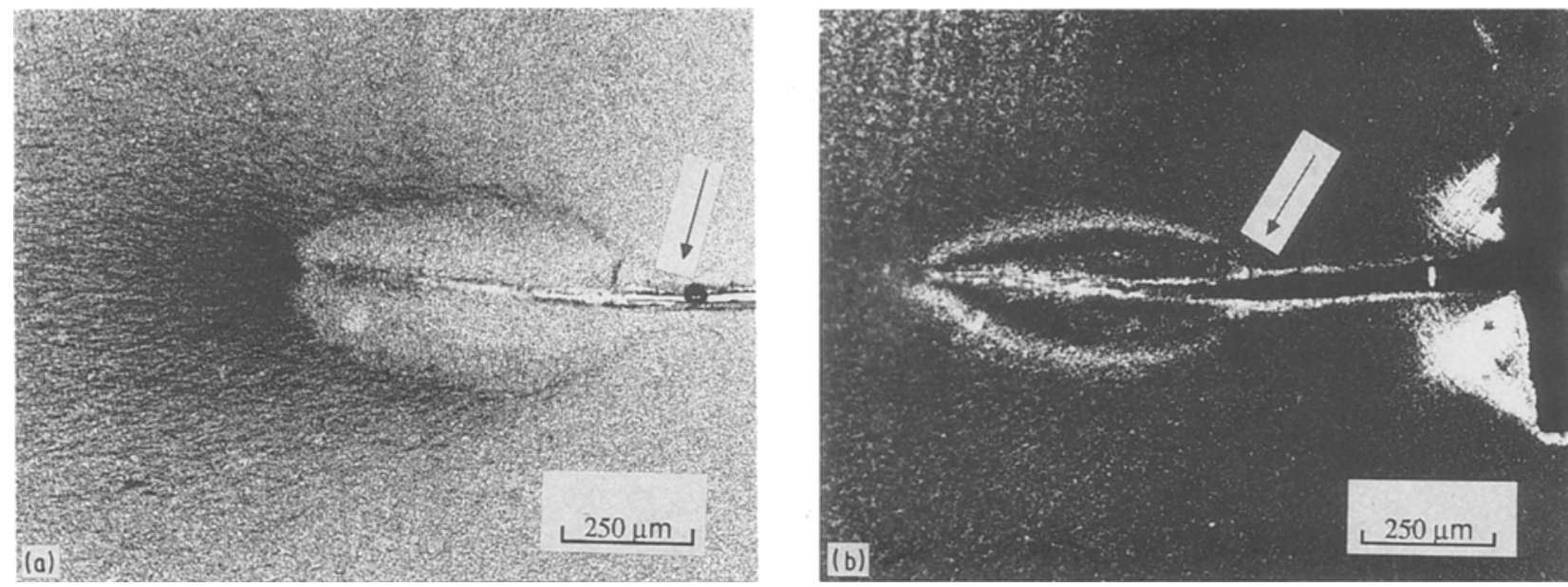

Figure 10 Transmitted light micrographs taken (a) in bright field and (b) between crossed polarizers at the SFSZ of DN-4PB specimen. The features are similar to those observed in Fig. 6 for the SEN-3PB specimen. A crazed zone is formed ahead of the crack tip. When the crack propagates through the crazed zone, the material is transformed into shear bands. The arrow indicates the tip of the starter crack.

lamella morphology of the elastomeric phase within the PPO is quite likely an ABA-type block copolymer with an unsaturated elastomeric mid-block, judging by the fact that staining by $\mathrm{OsO}_{4}$ was effective. Therefore, it appears that the block copolymer adheres well to the PPO phase. Thus, in our studies of toughening mechanisms we assume that excellent adhesion exists between the phases. At sufficiently low temperatures, the yield strength of the constituents increases to a level exceeding the adhesive strength between PA and PPO, and adhesive failure occurs, with relatively
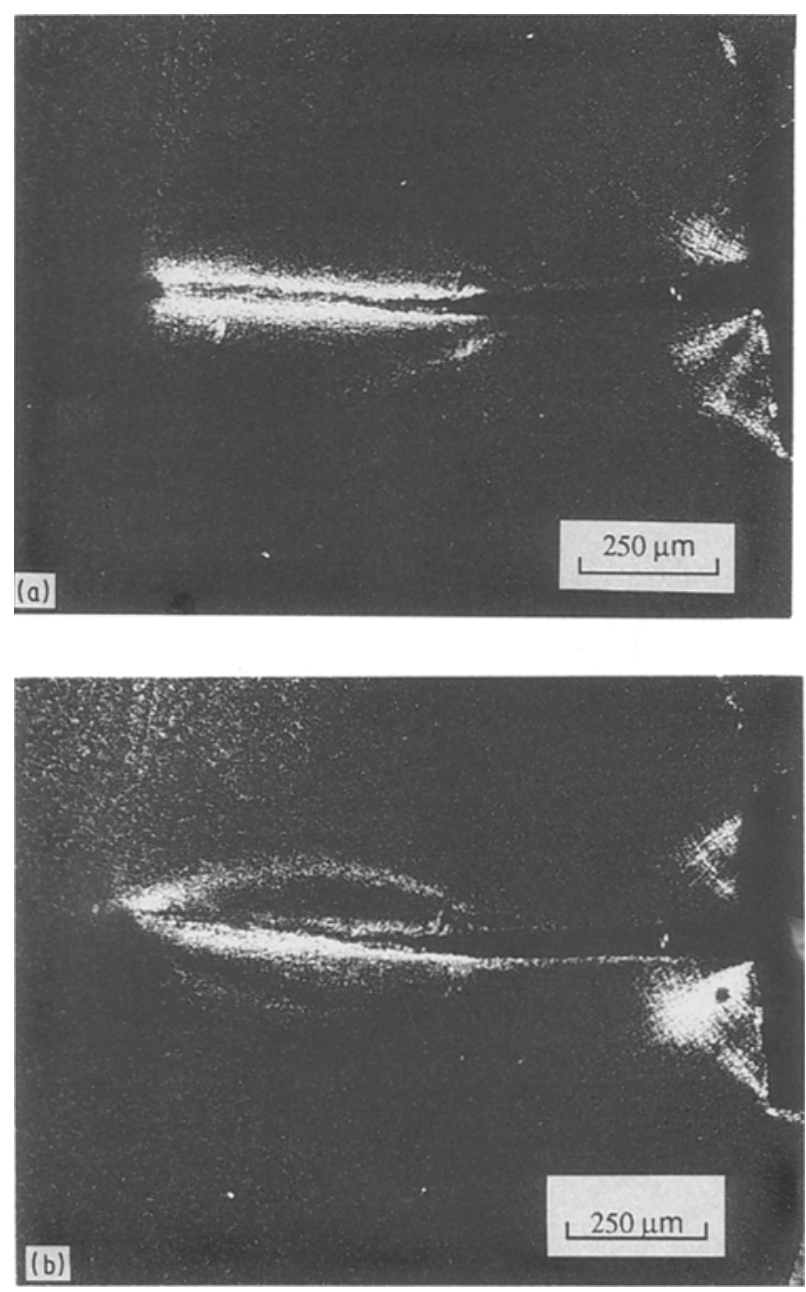

little plastic deformation to contribute to the toughness (Fig. 16).

Unfortunately, the tensile dilatometry studies and tapered tensile specimen experiments by themselves were only marginally useful for revealing the sequence of deformation events. Only the abrupt change in the volume dilatation curve (at $\sim 8.5 \% \varepsilon_{1}$ ) has been specifically correlated to the formation of crazes in the PA phase at $6000 \mathrm{psi}(40 \mathrm{MPa})$. No other form of cavitation is found. The complex dilatational behaviour of PA/ PPO from $\sim 1 \%$ to $\sim 3 \% \quad \varepsilon_{1}$ range cannot be corroborated by microscopic evidence. The initial increase in volume at $\varepsilon_{1}$ less than $1 \%$ is probably homogeneous and due to the Poisson's ratio effect. The calculated apparent Poisson's ratio is 0.45 which is a reasonable value for an injection-moulded PA specimen loaded in the flow direction. The PA phase probably contains an equilibrium amount of water $(\sim 3 \mathrm{wt} \%)$. The levelling off of the volume strain between 1 and $2 \% \varepsilon_{1}$ is probably due to homogeneous anelastic deformation. The softening of the $\sigma-\varepsilon$ curve in the range supports this

Figure /I Transmitted light micrographs taken between crossedpolarizers of the SFSZ of DN-4PB specimen. As the specimen is rotated from: (a) $-45^{\circ}$ (or $+45^{\circ}$ ), (b) $-10^{\circ}$, and (c) $+10^{\circ}$, with respect to the orientation shown in Fig. 10b, the birefringent pattern changes within the shear yielded zone. This shows that the featureless zone inside the SFSZ has also shear yielded.

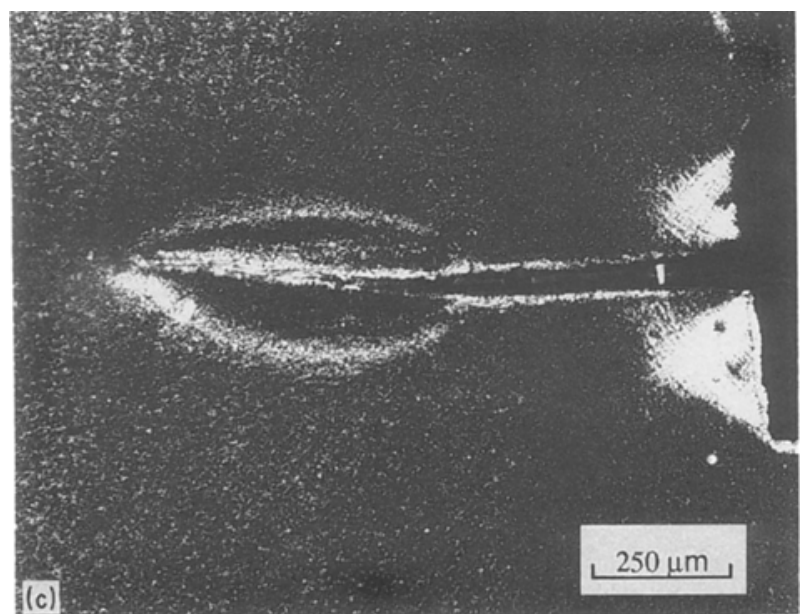



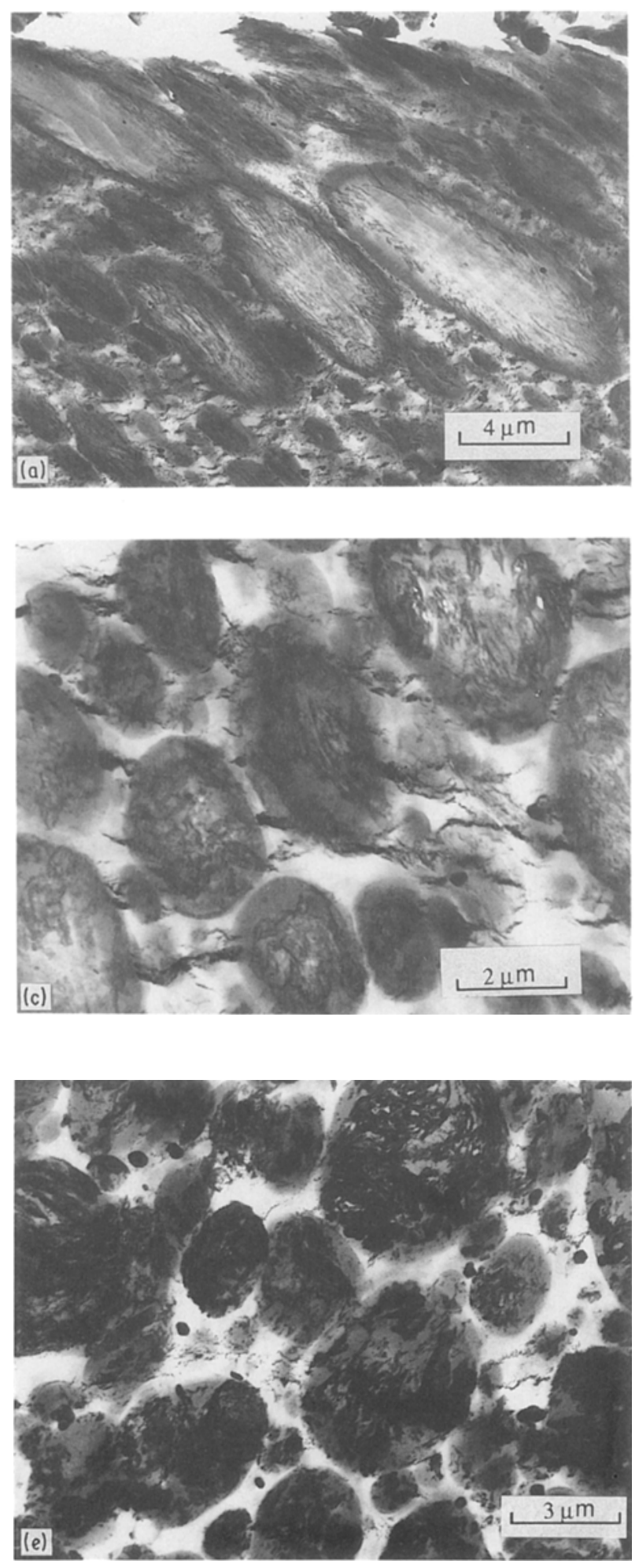

interpretation. Another possibility is that the PPO particles have caused sufficiently large stress concentrations in the PA matrix to initiate shear localization. The latter possibility is distinctly less likely to occur than the former one since the elastic moduli of PA and the elastomer-modified PPO are not significantly different. The volume increase at $\sim 2.5 \% \varepsilon_{1}$ is probably due to the initiation of crazes inside the PPO particles. Previous experiments have indicated that elastomermodified PPO begins to exhibit massive crazing at this strain range under similar testing conditions [27]. It is somewhat surprising that crazing in the PA phase does not occur until $8.5 \% \varepsilon_{1}$. The situation is complicated by the complex composition of the PA/PPO alloy. In
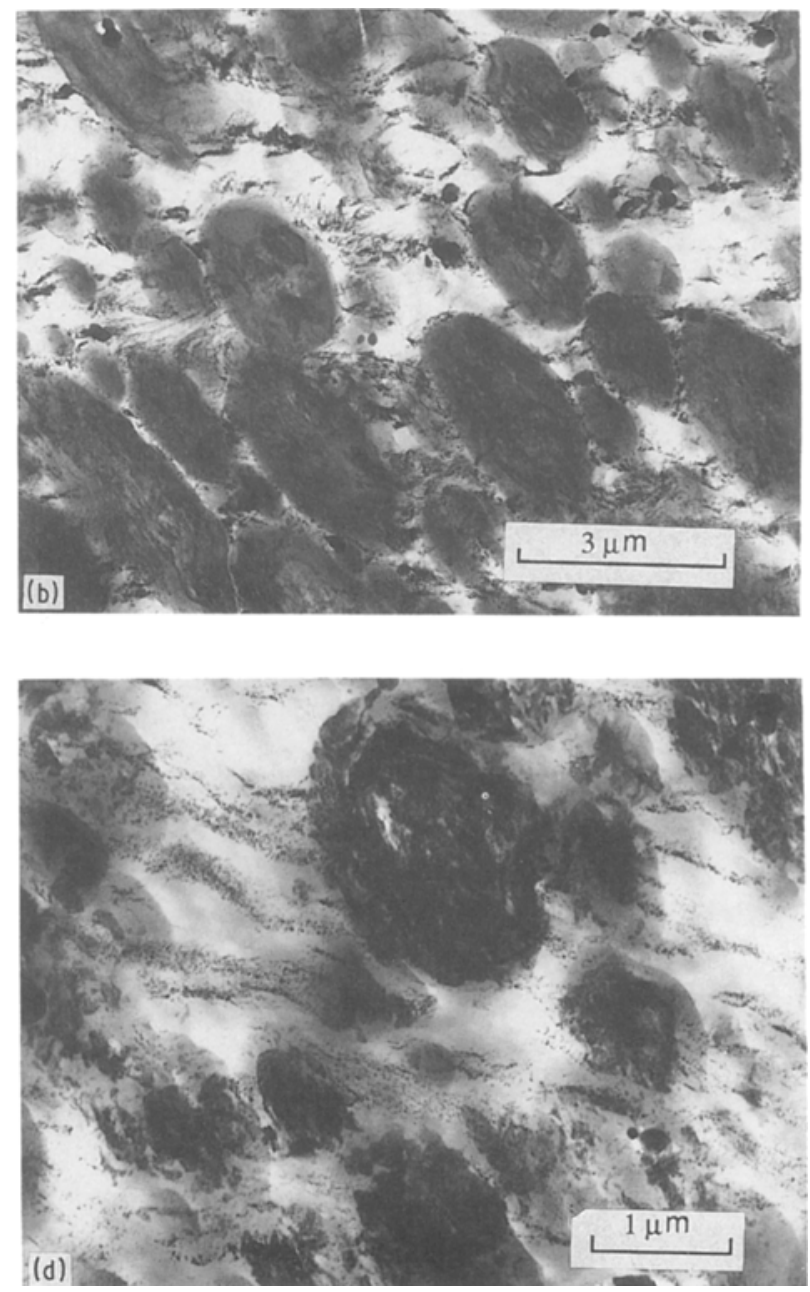

Figure 12 TEM micrographs of a DN-4PB thin section obtained from various regions around the crack. In (a), highly deformed PPO particles were formed immediately beneath the crack surface; in (b), the region farther beneath the fracture surface, the crazes appear to be somewhat closed or distorted; in (c), at the crazed zone, the crazes appear to be similar to those in the SEN-3PB samples; in (d), the crazes in front of the crack tip are rather diffuse; in (e), at the tip of the crazed zone farthest away from the crack tip, the PPO particles remain undeformed, the crazes appear to have just been nucleated. The crack propagates from right to left.

the TEM studies, the individual PPO particles appear to contain various amounts of the elastomeric phase. The inhomogeneous dispersion of the elastomeric phase in PPO causes various PPO particles to deform very differently, even at the same stress rate. Our future efforts include a study of PA/PPO alloys of controlled composition and morphology, which perhaps will allow us to account for all of the anomalous dilatational behaviours of this system. It is also quite likely that the strain rate employed in the tensile experiments was too low to indicate what might occur at the crack tip during the stable crack growth. An implicit assumption made in using tensile dilatometry to study toughening mechanisms is that the deformation mechanisms do not differ qualitatively in a uniaxial tensile specimen and at the crack tip of a SEN$3 P B$ or a DN-4PB specimen, even though the stress states are quantitatively different for these two types of tests. In the past this assumption has worked well in toughened styrenics [13], in polycarbonate/polyethylene blends [24], and in toughened epoxies [14] at 


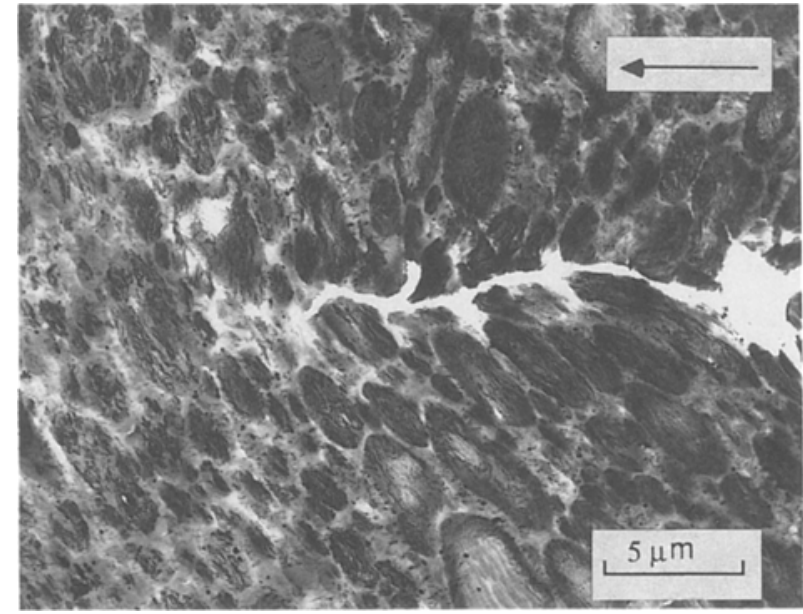

Figure 13 An TEM overview of the DN-4PB crack tip region. The PPO particles appear to deform at an angle with respect to the crack propagation direction. The arrow indicates the crack propagation direction.

high strain rates. In this last system, low strain rate results were found to be misleading. In the present study we performed tensile dilatometry studies at various strain rates ranging from $0.001 \mathrm{sec}^{-1}$ to $0.1 \mathrm{sec}^{-1}$, but the results do not differ much qualitatively and so we have concentrated our efforts on obtaining duplicate tests at the highest strain rate possible.

In the studies with the SEN-3PB specimens, valid $K_{\mathrm{lc}}$ or $G_{\mathrm{Ic}}$ values were not obtained due to ductile failure of the specimens. Studies on the SFSZ of the fractured specimen revealed that massive crazing and massive shear yielding are operative inside the damage zone. A study using DN-4PB specimens was employed to determine how the shear yielding and crazing mechanisms occur and interact with each other.

When the TOM and TEM micrographs taken from the SEN-3PB specimen are compared with those taken from the DN-4PB specimen, similar features are found. Therefore, the processes that occurred in the crack that stopped in the DN-4PB specimen are most likely the same as those in the crack that propagated unstably in the SEN-3PB specimen. In the DN-4PB specimen, it is worth noting that at least at the initial stage, the two notches experience similar, if not the same, stress states. Once one of the cracks propagates, each crack will experience a different stress state. Nevertheless, this type of experiment may offer critical information concerning events at the crack initiation and propagation stages. With these facts in mind, the
DN-4PB experiments turned out to be very effective for studying the toughening mechanisms of polymers.

In the DN-4PB experiment, one crack propagated from the starter crack upon loading and stopped when the second crack's growth became unstable and fractured. From Fig. 10, it is clear that the crazed zone is formed prior to crack propagation. As the crack propagates, the crazed zone is transformed into a shear yielded zone. The fact that a birefringent zone of uniform orientation was not observed in the shear yielded region can be rationalized as follows: in the stationary crack tip plastic zone, the plane of maximum shear stress is oriented in different directions; as a result, the material around the crack shear yielded along different orientations. Numerical simulation as well as experimental verification will be conducted in the future to justify the above statement.

The phenomenon of the transformation of a crazed zone into a shear yielded zone has never been observed experimentally. We now discuss possible reasons for its occurrence. Crazing itself is a cavitational process which can dissipate dilatational strain energy. On the other hand, if the extension of craze fibrils becomes too large, a crack may propagate through the craze and cause the material to undergo catastrophic failure. To prevent this from occurring the crazes must be stabilized, namely, prevented from experiencing excessive extension. In the PA/PPO alloy, the crazing mechanism itself, which appears to be stable, is apparently sufficient to relieve the hydrostatic tension and allow the shear yielding mechanism to be activated. These findings are consistent with the toughening mechanism we proposed previously [14], which suggests that the triaxial tension at the crack tip must be dissipated in order for the remaining deviatoric stress to reach a critical value for yielding. The elastomeric phase inside the PPO particles undoubtedly plays an important role. It initiates the crazes inside the PPO particle, which then propagate from the PPO particles into the PA matrix. When the stress state favours the formation of shear bands, the elastomeric phase, as a stress concentrator, causes the PPO particles to shear yield more readily. This event, in turn, triggers the bulk shear yielding of the PA matrix şince a yielded particle is mechanically equivalent to a very soft particle. The PA/PPO alloy is then toughened. The above statement agrees with the findings of our finite element methods analysis $[1,28]$.

The opening near the crack tip seems to be compressed (see Fig. 10). This phenomenon may be

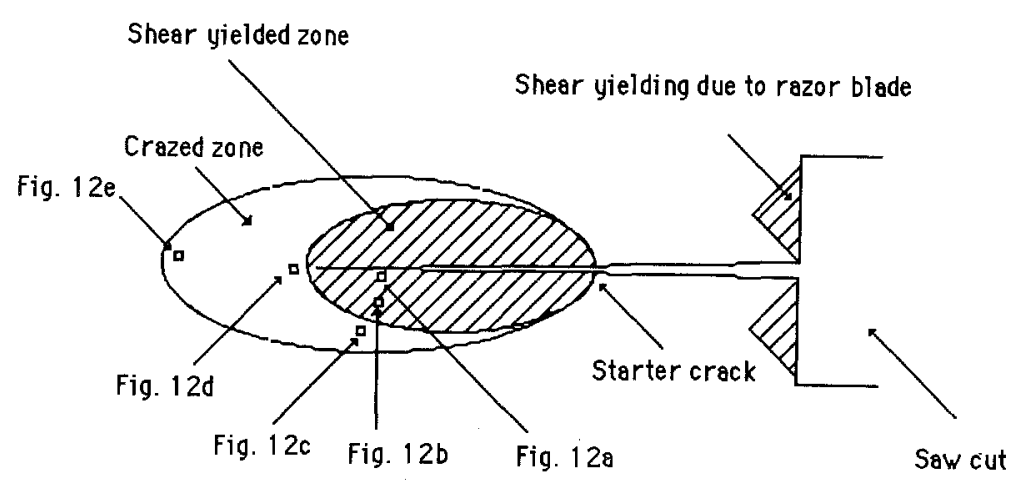

Figure 14 A schematic drawing of the deformation features in the damaged DN-4PB specimen. The location of TEM micrographs shown in Fig. 12 are indicated in this drawing. Note that the features are not drawn to scale. 


\section{Ea : Crazing \\ 国: Shear yielding}

(a)

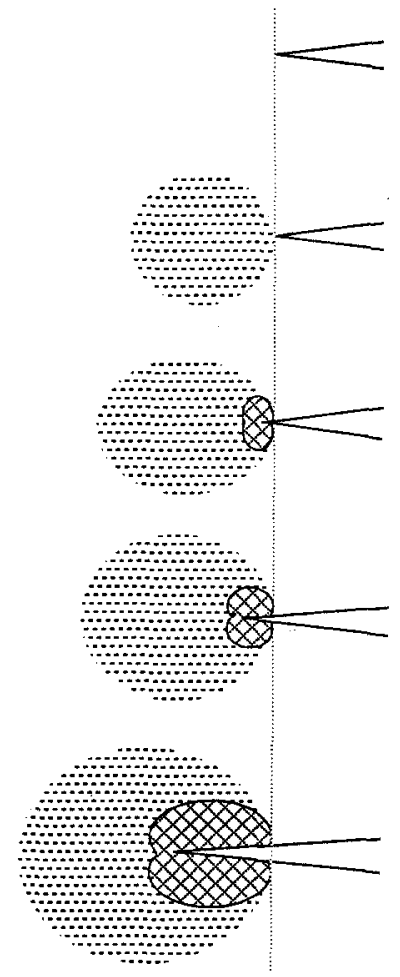

Figure 15 A sketched sequence of the toughening mechanisms. (a) The initial starter crack; (b) formation of a crazed zone in front of the crack tip when the specimen is initially loaded; (c) formation of initial shear yielded plastic zone around the crack tip when the hydrostatic tension is relieved due to the formation of crazes; (d), once the build-up of shear strain energy reaches a critical value, the material begins to undergo shear yielding, and the crack propagates; (e), a damage zone surrounds the propagating crack before the crack grows unstably.

rationalized by the so-called zone shielding mechanism [29]. Zone shielding in the PA/PPO alloy is possible due to the existence of the craze zone in the wake of the SFSZ. These crazes, similar to the microcracks formed in toughened ceramics, invariably form in regions of residual tension and, thereby, result in an

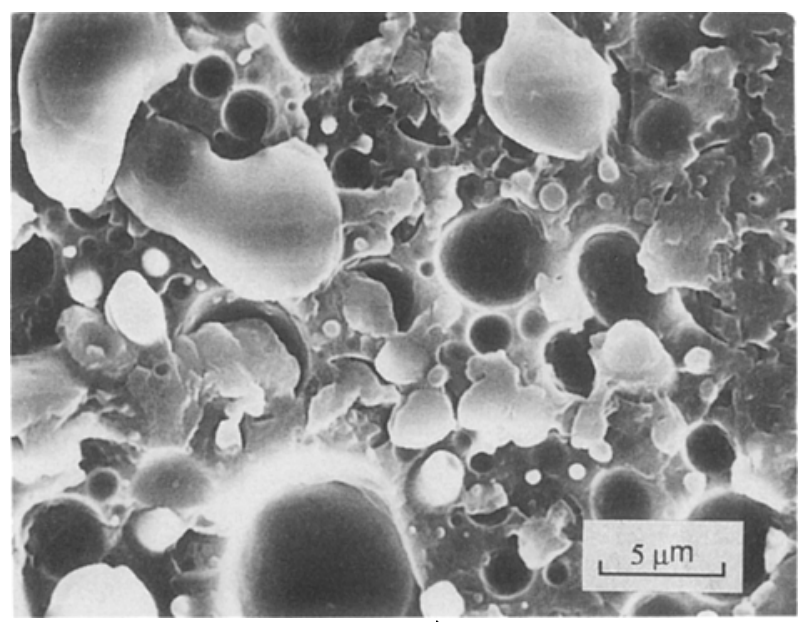

Figure 16 SEM micrograph of a notched specimen impact fractured at a low temperature. The fracture surface indicates that only very limited plastic deformation has occurred. Adhesive failure between the PPO and the PA phase is observed. The crack propagates from right to left. attendant dilatation [30-32]. As a result, the stress intensity at the crack tip is reduced [29]. This mechanism serves as an additional deterrent to crack propagation. The contribution of zone shielding to the overall toughness of a system depends on the size of the craze zone in the wake of the SFSZ [31]. The closing-up of the crack tip also may be attributed to elastic recovery of the material after unloading. Our current research will verify this mechanism.

A crack tip blunting mechanism [33-35] also appears to be operative. The TEM investigation of the DN-4PB specimen (see Fig. 13) confirms that the PPO particles were highly elongated around the crack tip. The large deformation of the PPO particles suggests that plastic deformation (shear yielding) has occurred. As a result, the crack tip may have been effectively blunted by the surrounding plastically deformed material. Again, as stated earlier, in order to have shear yielding, the crazing mechanism must be operative prior to the formation of shear yielding. Consequently, both crazing and shear yielding must be present in order for the crack tip blunting mechanism to be effective in the PA/PPO alloy.

\section{Conclusion}

Toughening mechanisms of a commercial PA/PPO alloy have been examined using a variety of techniques. The results confirm that the PA/PPO alloy is toughened first by nucleation of a crazed zone ahead of the crack tip and, as the crack propagates, by transformation of the crazed zone into a shear yielded zone. The elastomeric phase plays an important role in these mechanisms, as well. Finally, TOM and TEM studies suggest that crack shielding and crack blunting are also causes of the observed increase in toughness of the PA/PPO alloy.

The current research emphasizes the qualitative aspects of the toughening mechanisms in a PA/PPO alloy. Investigations of other PA/PPO alloys of controlled compositions and morphology will be undertaken. With these studies, it should be possible to understand the individual role that each constituent plays in the toughening process. Quantitative measurements of the fracture toughness and studies of the possible zone shielding and crack blunting mechanisms will also be conducted.

\section{Acknowledgements}

This work is partially supported by a Material Research Group grant from the National Science Foundation, No DMR-8708405. The authors would like to thank Miss Lisa Maison for her help with the TEM work.

\section{References}

1. A. F. YEE, D. S. PARKER, H. J. SUE and I-C. HUANG, Proceedings of the PMSE Div., 194th American Chemical Society National Meeting (August, 1987).

2. K. KOO, T. INOUE and K. MIYASAKA, Polym. Eng. Sci. 25 (1985) 741

3. T. KURAUCHI and T. OHTA, J. Mater. Sci. 19 (1984) 1699.

4. H. J. SUE and A. F. YEE, "Toughening Mechanism(s) in Alloys of Rigid Polymers" (The Society of Plastics Engineering, Chicago, 1987).

5. A. S. WOOD, Modern Plastics, April (1985) 81. 
6. K. MIN and J. L. WHITE, Polym. Eng. Sci. 24 (1984) 1327

7. A. GHAFFAR, C. SADRMOHAGHEGH and G. SCOTT, Europ. Polym. J. 17 (1981) 941.

8. C. SADRMOHAGHEGH, G. SCOTT and E. SEROUDEH, Europ. Polym. J. 19 (1983) 19.

9. M. E. J. DEKKERS and S. Y. HOBBS, Polym. Eng. Sci. 27 (1987) 1164

10. Y. FUJITA, K. KOO, J. ANGOLA, T. INOUE and T. SAKAI, Kobunshi Ronbunshu 43 (1987) 119.

11. R. R. DURST, R. M. GRIFFITH, A. J. URBANIC and W. J. VAN ESSEN, ACS Div. Org. Coat. Plast. Prepr. 34 (1974) 320.

12. A. M. DONALD and E. J. KRAMER, J. Appl. Polym. Sci. 27 (1982) 3729.

13. C. B. BUCKNALL, "Toughened Plastics" (Applied Science, London, 1977).

14. (a) A. F. YEE and R. A. PEARSON, J. Mater. Sci. 21 (1986) 2462. (b) R. A. PEARSON and A. F. YEE, ibid. 21 (1986) 2475.

15. A. J. KINLOCH, Proceedings of the PMSE Div., 194th American Chemical Society National Meeting (August, 1987).

16. W. BASCOM and R. TING, J. Mater. Sci. 16 (1981) 2657.

17. J. SUltan and F. McGARRY, Polym. Eng. Sci. 13 (1973) 19.

18. M. H. HAHN, R. W. HERTZBERG and J. A. MANSON, J. Mater. Sci. 21 (1986) 39.

19. S. WU, Polymer 26 (1985) 1855.

20. R. BORGGREVE, R. GAYMANS, J. SCHUIJER and J. I. HOUSZ, ibid. 28 (1987) 1489.

21. F. RAMSTEINER and W. HECKMANN, Polym. Commun. 26 (1985) 199.

22. H. BREUER, F. HAAF and J. STABENOW, J. Macromol. Sci.-Phys. B14 (1977) 387.
23. S. CIMINO, F. COPPOLA, L. D'ORAZIO, R. GRECO G. MAGLIO, M. MALINCONICO, C. MANCARELLA E. MARTUSCELl and G. RAGOSTA, Polymer 27 (1986) 1874

24. M. A. MAXWELl and A. F. YEE, Polym. Eng. Sci. 21 (1981) 205 .

25. A. S. HOLIK, R. P. KAMBOUR, S. Y. HOBBS and D. G. FINK, Microstruct. Sci. 7 (1979) 357.

26. N. SHIBUYA, European Patent Application, 0225170 AI, 1986.

27. A. F. YEE, unpublished work

28. H. J. SUE and A. F. YEE, Polymer, submitted.

29. R. O. RITCHIE and W. YU, in "Small Fatigue Cracks", edited by R. O. Ritchie and J. Lankford (TMS-AIME, Warrendale, $P A, 1986$ ).

30. R. A. SCHMIDT and T. J. LENTZ, ASTM STP 678 (American Society for Testing and Materials, Philadelphia, 1979) p. 166

31. A. G. EVAns and K. T. FABER, J. Amer. Ceram. Soc. 67 (1984) 255 .

32. A. G. EVANS and R. M. CANNON, Acta Metall. 34 (1986) 761

33. J. G. Williams and J. M. HODGKInSON, Proc. $R$. Soc. A375 (1981) 231.

34. A. J. KINLOCH and J. G. WILliAMS, J. Mater, Sci. 15 (1980) 987.

35. R. A. GLEDHILL and A. J. KINLOCH, Polym. Eng. Sci. 19 (1979) 82.

Received 16 February

and accepted 14 June 1988 